\title{
CASE
}

OF

\section{FUNGUS HÆMATODES}

OF THE

\section{BRA I N.}

\author{
By JOHN HUNTER, EsQ. JuN. \\ MEMBER OF THE ROYAL COLLEGE OF SURGEONS. \\ COMMUNICATED \\ By Mr. GREEN.
}

Read June 23rd, 1824.

MISS M. A. was afflicted with severe head-ache in the early part of 1820 , being then in her seventeenth year. She was of a delicate frame, "light hair and eyes, fair complexion, and of a mild and cheerful disposition. She had previously enjoyed good health, menstruated regularly, had not received any blow or injury, and knew of no cause to which the complaint could be assigned. Common means afforded relief, and she went down into Cheshire for four months during the summer, where she was in the habit of taking daily exercise, and on one occasion walked ten miles without much inconvenience, but was never entirely free from head-ache. Shortly after her return to town the pain again became very distressing, and she 
again derived benefit from the medicines administered, and the application of a blister to the neck. In January 1821, in consequence of a severe relapse of pain, leeches were applied to the forehead, after which she had a long interval of comparative ease. In February she was at a ball, danced for several hours, and appeared to enjoy herself much ; nor did she apply for further advice, till the 30th of May following. Her symptoms were then much aggravated, and she became rapidly worse. The pain of the head assumed a more serious character. It was usually referred to the right temple, and she experienced a regular exacerbation every morning, to such a degree, that she would roll about the bed in agony for an hour or two, after which the pain gradually subsided, and continued more tolerable during the day. In addition to this, she was affected with vertigo, occasional syncope, great dread of imaginary objects, a state of high nervous irritation, dullness of hearing, and indistinct vision. She became short sighted; objects appeared larger than natural, and at times she was totally blind for several seconds. Along with these symptoms she had quickness of pulse, heat of skin, violent pain in the stomach, sickness and vomiting. Very severe metastatic pains, unattended with any external appearance of inflammation, attacked in succession various parts of the body, at one time the throat, occasioning extreme diffculty of deglutition, at another the chest, impeding the respiration, at another different parts of the spine (more particularly towards the neck), 
the knees, the ancles, and the wrists. Blisters, cold applications to the head, mercury in small doses, though not to the extent of salivation, and a variety of other means were tried, but with little or no relief. Her health declined fast, and she became much emaciated from the constant vomiting.

On the 31st of August, 1821, she was attacked, while in bed, with a fit of strong convulsions. The whole of the body was thrown into violent action, attended with strabismus and screaming. This lasted about half an hour, and when the struggling ceased she was left in a state of stupor; from which, however, she recovered during the night. On my visit the following day, I found her condition materially altered for the worse. She had now lost all power over the body, and could not raise herself or even turn from side to side in the bed; her legs and arms she could move, but with less freedom; her sight, which though imperfect had hitherto enabled her to discern objects, was now so far lost that she could only perceive the difference between light and darkness when the change was sudden. The pupils were much dilated and slightly affected by the light. Her deafness also was greatly increased, and she could with difficulty catch any sound: The failure in the sight and hearing occurred first on the left side, being opposite to that in which the pain was originally fixed. The bowels were obstinately costive; the vomiting and pain of the stomach continued; pain of the head intense; 
pulse quick, respiration hurried, skin hot and dry, sleep tranquil and without stertor. In the course of a few days she had a repetition of the same kind of fit; after which they returned with more or less frequency and severity, till within a short period of her death, generally influenced, however, by the state of the alimentary canal. Sometimes she had five or six in a day, and occasionally she would pass several days without any fit. They usually came on without warning, but sometimes they appeared to be produced by slight exertion. Besides these general convulsive attacks, she was subject to spasmodic twitchings, and startings of different parts of the body. Her sight and hearing were soon lost altogether; the smell was also entirely lost, and the taste, if any remained, was very imperfect. She expressed a desire for particular articles of food, but always complained of their being insipid, and could seldom tell what she was eating.

Being deprived of all the organs of sense connected with the brain, the only mode of communication that could be devised, was the common method of talking with the fingers, the person with whom she was conversing indicating each letter upon her fingers. She was soon able to distinguish, by the feel, every person with whom she was in the habit of talking, and acquired considerable facility in this way of conversing, guessing the words before they were half spelt. She would thus keep one or other of her attendants constantly 
employed when awake. She was anxious to amuse herself with some kind of manual occupation, but her arms were so feeble that she could not bear the fatigue of any that were tried. Her intellect was unimpaired, except when under the influence of the fits. She appeared to be aware of her hopeless condition, and desired that her head might be opened after her death. She evinced great patience under her sufferings, and was even cheerful when the pain was moderate; she was seldom however, when awake, free from intense pain in the head, of a lancinating or throbbing kind, not confined to any particular part. The pain at the upper and lower part of the spine, the sensation of extreme coldness down the back, pain in the right and afterwards in the left breast, were also at times exceedingly distressing. The face was often swelled, apparently œdematous, though at other times quite shrunk. The cheeks were subject to partial flushings; the eyes retained their lustre; the pupils were fully dilated, and quite insensible to light. The heat of the skin was frequently very oppressive, and the itching at times intolerable. She rarely complained of cold, excepting down the spine. The tongue was occasionally furred, but generally clean; no thirst. The appetite, after the vomiting ceased, became almost insatiable, and she recovered flesh.

Subsequently she had repeated attacks of bilious vomiting, reducing her each time to a state of excessive debility, from which she as often rallied 
in a surprising manner. The vomiting was apparently produced by the action of the stomach alone, unassisted by the abdominal muscles. The bowels were obstinately torpid, seldom acting without the aid of cathartics. She once went fourteen days without an evacuation. Her symptoms were invariably aggravated when the bowels were long confined. The urine was passed involuntarily during the fits, but was at other times under the influence of the will. The catamenia had not appeared since she had been confined to her bed; respiration natural and easy; speech unaltered; voice clear and distinct; pulse varying from 80 to 100 , small, and generally weak; sleep very easy and undisturbed, except by her crying out to be turned, after which she would doze off again directly. She could lie on her back, or on either side, but was unable to rest in the same position above half an hour at a time, so that she required some person constantly in attendance to turn her, and if it was not done as soon as asked for, she frequently went into a fit. She never recovered the power of her body, nor could she move her head in the least degree, but her sensation was quite perfect. Several attempts were made to raise her gradually in the bed, but they always produced considerable pain, and, if persisted in, brought on a fit. The medicines exhibited were merely intended to relieve her sufferings, except that an attempt was made to affect the system with mercury; but the fits increased so much during its use, that her mother requested it might be discontinued. 
The above symptoms continued with more or less urgency till February 1823, when her powers began to fail altogether, the stomach rejecting every kind of food. No evacuation could be procured from the bowels, without the aid of injections; the whole muscular system seemed to lose its tone; the limbs were drawn into a semiflexed position, and she had scarcely strength to move them; the lips were half closed, the meuth full of aphthous ulcerations, and the teeth covered with sordes; the features were distorted; she slept with her eyelids half open; the eyes became dim; inflammation came on in the left eye, which proceeded to ulceration and opacity of the cornea. She expressed no pain, and was not even aware that the eye was affected. The urine and fæces were passed involuntarily. . She could not swallow any food unless it was reduced to a liquid form, and then only with difficulty. She had a troublesome cough, which, from her extreme debility, frequently threatened suffocation; it seemed to be occasioned by mucus irritating the upper part of the larynx. Pain in the head continued to distress her, but the fits were less frequent, and appeared incapable of producing the same convulsive action, for want of power in the muscles; and she did not require to be turned so often. Her faculties also declined; she talked very little, and only of her complaints. Her pulse was so feeble as to be scarcely perceptible. She still breathed freely, and slept much, and remarkably easy. The composed sleep which she enjoyed from the com- 
mencement of the disorder, had always proved a source of great consolation to her friends.

In September, a slight diarrhoea came on : she could then scarcely take any sustenance, and had become so much emaciated that the skin was excoriated in several places, from the pressure of the bones. She died, apparently without much suffering, in a state of complete exhaustion, on the 5th of October, 1823, having lingered more than two years from the first attack of convulsions, and nearly four years from the commencement of the head-ache.

This interesting case was attended in the early part by Drs. Babington and Birkbeck; was seen repeatedly during its progress by Mr. Cartwright, Surgeon to the Middlesex Hospital; once by $\mathrm{Mr}$. Green, of St. Thomas's Hospital ; and by one or two other medical gentlemen, besides my father and myself, who visited her regularly from the first of her illness.

I inspected the body the day after her decease, in the presence of Mr. Cartwright and Mr. Yarnold. The following appearances were remarked.

The scalp was slightly œdematous. The bones of the cranium extraordinarily thin, and several short spicula projected inwards, from the posterior part of either parietal bone. The membranes covering the brain were free from disease; the sub- 
stance of the cerebrum rather softer than usual; from eight to ten ounces of fluid in the ventricles; the membrane lining the ventricles of a dingy yellow colour. The thalami nervorum opticorum were somewhat enlarged, irregular on their surface, and entirely converted into the fungous disease. A longitudinal section through one of the thalami presented exactly the appearance of a portion of coagulated blood. The corpora striata were not affected, but the disease extended into the adjacent parts of the cerebrum and cerebellum below, and also to the lower and posterior edge of the falx major. The optic nerves were of a darker colour than usual, but did not appear to be altered in texture. The other cerebral nerves presented no deviation from their natural structure. The spinal marrow, as far as could be traced through the foramen magnum, was perfectly healthy. There were several sharp ridges of bone at the basis of the cranium, and the irregularities were all very strongly marked.

The viscera of the thorax and abdomen were carefully examined throughout, but no diseased appearance was found, except a number of small biliary concretions about the consistence of wax, and a quantity of inspissated bile lodged in the gall bladder. The diseased portion of brain, was presented to Mr. Green, and is now in the museum of St. Thomas's Hospital. 


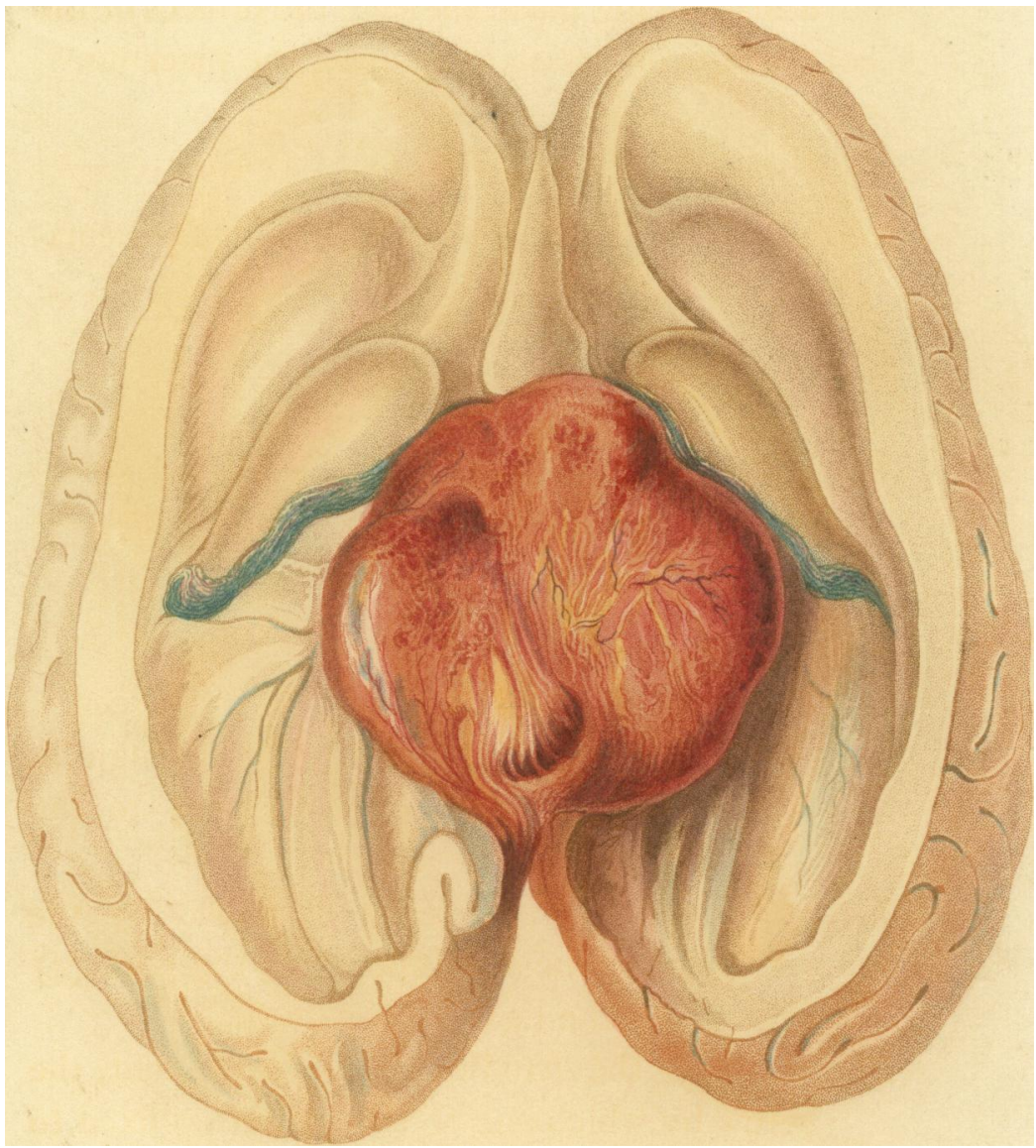

